\title{
The Incidence of Chromosomal Aberrations in Anaesthetists
}

\author{
Flossie Jayakaran and I. Manorama Thomas \\ Division of Human Genetics, Department of Anatomy, St. John's Medical College, Bangalore, India
}

Accepted November 14, 1997

\begin{abstract}
Summary Anaesthetists have reported complaints of headache, nausea, fatigue, dizziness, as well as heart and liver diseases as a consequence of chronic exposure to low ambient levels of anaesthetic gases in the operation theater. Reports of foetal wastage, bad obstetric history and infertility have also been recorded. This study was amined at evaluating the damage to chromosomes by quantifying the damage in terms of the number of chromosomal aberrations (CAs) seen when compared to equally matched controls. The study showed that the anaesthetists showed a significantly higher number of chromosomal aberrations than did the controls. This is evidence of genotoxicity, and suggestions are made to reduce the exposure in the theater by the installation of efficient scavenging systems and leak proof ventilation systems on a top priority basis.
\end{abstract}

Key words Chromosomal aberrations, Volatile anaesthetics, Halothane, Genotoxic.

Anaesthesia is a regular feature in all surgical interventions. Attempts to keep the operation theater (OT) sterile, leads to an accumulation of waste anaesthetic gases (AGs), escaping from the anaesthetic apparatus as well as exhalations from the anaesthetized patients. With the passage of time and in the absence of scavenging systems, there is a significant build-up of the gas levels in the theater.

Various complaints from OT personnel have been attributed to chronic inhalation during surgery of the ambient AGs in the theater. The AG levels are also higher in the area immediately surrounding the anaesthetist (Spierdijk and Burm 1982).

Epidemiological studies have ascribed adverse reactions by anaesthetists as a consequence of exposure. These reported adverse effects range from feelings of discomfort, headache, nervousness, sleep loss, fatigue, pruritus, increased cancer rate, hepatic and renal diseases to reports of infertility, increased rates of abortions, miscarriages and congenital abnormalities in children born to practicing anaesthetists, as well as a sex ratio favouring the female sex. The aim of the present work was to investigate the toxicity of AGs on the chromosomes of anaesthetists for the incidence of chromosomal aberrations (CAs) like breaks, gaps, rings, dicentrics and exchanges. CAs are very sensitive indicators of mutagenicity and the residual aberrations left behind (after normal cellular repair), remain dormant until stimulated to divide (Bloom et al. 1966). The subjects chosen for this report had had chronic exposure to low levels of AGs as they worked in OTs which did not possess a scavenging system except for air-conditioners or fans.

\section{Materials and methods}

$4 \mathrm{ml}$ of blood per subject was collected in heparinised syringes, from 51 anaesthetists $(31 \mathrm{men}$ and 20 women), ranging in age from $28-60$ years, who were in active service. Their exposure to anaesthetics ranged from 2 years to 29 years.

The controls chosen were matched with the subjects for sex, age ( \pm 1 year), diet, smoking habits and lifestyle, from among the physicians who were not exposed to AGs. Smoker subjects

Address for correspondence: Flossie Jayakaran, Dept. of Anatomy, St. John's Medical College, Bangalore 560034 , Karnataka, India. e-mail: chrisj@giasbg01.vsnl.net.in 
were matched with controls who smoked the same number of cigarettes per day.

Both subjects and controls were healthy, were not on antibiotics and provided medical and obstetric histories.

The cultures were set up according to the technique of Arakaki and Sparkes (1963) with minor modifications. The samples were set up for $48 \mathrm{hr}$. BrdU was added in the dark prior to incubation, to facilitate differentiation of M1 cycles from subsequent cell cycles. All procedures were made under sterile conditions. After termination, they were dropped on clean, cold wet coded slides, air dried, aged and stained by the FPG staining method (Perry and Wolff 1974), for differential staining of sister-chromatids. $100 \mathrm{M} 1$ metaphases (1st cycle metaphases) were scored for aberrations in each subject and control as per the scoring criteria given in ISCN 1985. The results obtained were subjected to statistical evaluation.

\section{Results}

\section{Chromosomal aberrations}

The metaphase spreads were scored for the following structural chromosomal aberrations: chromatid gaps and breaks, chromosome gaps and breaks, exchanges, dicentrics, double minutes, ring chromosomes etc. A significant difference was seen in the number of aberrations in subjects compared to controls (Table 1). All aberrations were significantly greater in anaesthetists than in controls. There were significantly more chromatid gaps (Chi square $=12.93, \mathrm{df}=1, \mathrm{p}<0.001$ ) (Fig. 1), chromatid breaks (Chi square $=6.47, \mathrm{df}=1, \mathrm{p}<0.01$ ) (Fig. 2), chromosome gaps (Chi square $=3.24, \mathrm{df}=1, \mathrm{p}<0.05$ ) (Fig. 3), dicentrics (Chi square $=7.80, \mathrm{df}=1, \mathrm{p}<0.01$ ) (Fig. 5), fragments and double minutes (Chi square $=23.99, \mathrm{df}=1, \mathrm{p}<0.001$ ). Other chromosomal aberrations seen were chromosome breaks (Fig. 4), ring chromosomes (Fig. 6) and endoreduplication (Fig. 7) which did not differ significantly from that seen in controls. Table 2 shows the distribution of the various kinds of chromosomal aberrations in subjects and controls.

Univariate procedures were used to examine the influence of different independent variables on the number of chromosomal aberrations. No significant relationship was seen for age $(r=0.11$, $\mathrm{df}=49)$, years of exposure $(\mathrm{r}=0.06, \mathrm{df}=49)$, or exposure in $\mathrm{hr} /$ day $(\mathrm{r}=-0.03, \mathrm{df}=49)$.

The sex of the subject seemed to affect the number of chromosomal aberrations with male subjects showing more chromosomal aberrations than female subjects $(4.81 \pm 3.04$ vs. $3.50 \pm 1.82$, $\mathrm{t}=1.92, \mathrm{df}=49, \mathrm{p}=0.06$ ). The influence of smoking on chromosome aberrations also showed a trend towards significance (non-smokers $\mathrm{n}=44,4.55 \pm 2.65$, smokers $\mathrm{n}=7,2.71 \pm 2.56, \mathrm{t}=1.71$, $\mathrm{df}=49, \mathrm{p}=0.094)$.

\section{Discussion}

The adverse effects of AGs in anaesthetists (as evidenced by the significantly greater number of CAs when compared to controls) is believed to be due to the exposure of the anaesthetists to the accumulation of waste AGs in the OT. This accumulation is marked in theaters which do not pos-

Table 1. Showing aberrations in subjects and controls

\begin{tabular}{ccc}
\hline \hline & $\begin{array}{c}\text { No. of aberrations } \\
(\text { Mean } \pm \text { SD })\end{array}$ & Significance \\
\hline $\begin{array}{c}\text { Anaesthetists } \\
(\mathrm{n}=51) \\
\text { Controls } \\
(\mathrm{n}=51)\end{array}$ & $4.29 \pm 2.69$ & $\mathrm{t}=6.16, \mathrm{df}=50, \mathrm{p}<0.001$ \\
\hline
\end{tabular}




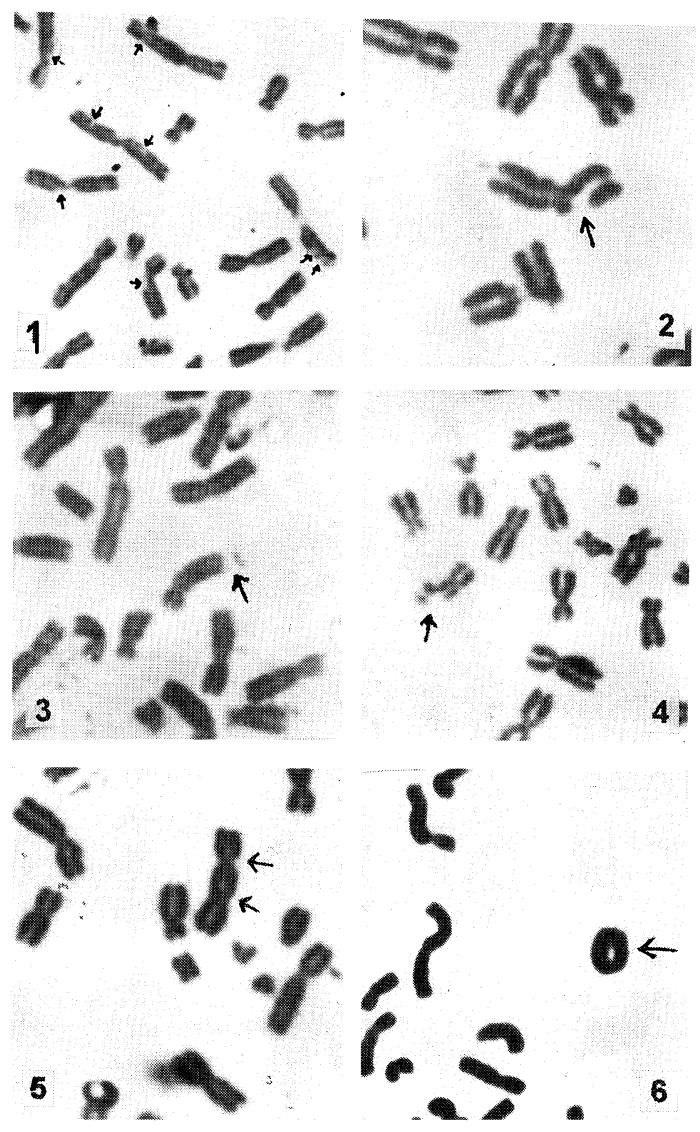

Figs. 1-6. Chromosomal aberrations seen in anaesthetists. 1. Chromatid gap (ctg), 2. chromatid break (ctb), 3. chromosome gap (csg), 4. chromosome break (csb), 5. dicentric (dic), 6. ring chromosome (r).

Table 2. Presence $(+)$ or absence $(-)$ of different CAs in the sample

\begin{tabular}{|c|c|c|c|c|c|c|c|c|c|c|c|c|c|c|c|c|c|c|c|c|}
\hline \multirow{2}{*}{ Aberrations } & \multicolumn{2}{|c|}{ ct gaps } & \multicolumn{2}{|c|}{ ct breaks } & \multicolumn{2}{|c|}{ cs gaps } & \multicolumn{2}{|c|}{ cs breaks } & \multicolumn{2}{|c|}{ Rings } & \multicolumn{2}{|c|}{ Dicentric } & \multicolumn{2}{|c|}{ Exchanges } & \multicolumn{2}{|c|}{ Tetraploidy } & \multicolumn{2}{|c|}{$\begin{array}{c}\text { Others } \\
\text { (frag }+ \text { min) }\end{array}$} & \multicolumn{2}{|c|}{$\begin{array}{c}\text { All } \\
\text { aberrations }\end{array}$} \\
\hline & + & - & + & - & + & - & + & - & + & - & + & - & + & - & + & - & + & - & + & - \\
\hline 51 Anaesthetists & 36 & 15 & 15 & 36 & 34 & 17 & 12 & 39 & 3 & 48 & 9 & 42 & 4 & 47 & 9 & 42 & 21 & 30 & 46 & 5 \\
\hline 51 Controls & 17 & 34 & 4 & 47 & 24 & 27 & 6 & 45 & 0 & 51 & 0 & 51 & 3 & 48 & 3 & 48 & 0 & 51 & 35 & 16 \\
\hline
\end{tabular}

sess a scavenging system, exposing the anaesthetists, surgeons, nurses, technicians and helpers to its possibly deleterious effects. Exposure concentrations are extremely variable, but may reach levels of atleast $50 \mathrm{ppm}$ for halothane and 5,000 ppm for nitrous oxide in unscavenged OTs (Baden and Simmons 1980).

The narcotic action of anaesthetics is due to its solubility in lipid cell membranes. The greater the solubility, the longer is its retention in the body, which in turn increases the time taken for elimination as well as its availability for biodegradation. This results in the accumulation of toxic and potentially mutagenic metabolites (Baden and Simmons 1980). Holmberg et al. (1982) suggests that AGs may act as a weak co-mutagen, which potentiates the genotoxic effects of other environmental factors, of which stress may be a prime candidate among anaesthetists. McNamee et al. 
(1987) suggested that stress, fatigue and exposure to AGs may be responsible for the higher numbers of ill-health retirements and/or deaths among anaesthetists.

Volatile anaesthetics may be exhaled/excreted by the lungs, but are also metabolized in vivo by the cytochrome P-45 (mixed function oxidase) system, the enzymes of which are located in the SER of the liver and kidney. Halothane gets metabolized oxidatively to trifluroacetic acid, bromine and chlorine. Trichloroacetaldehyde, an intermediary product is highly reactive and binds to tissue macromolecules. Bromochlorodifluoroethylene (BCD), another intermediary is a potent alkylating agent (Baden and Simmons 1980).

Nurse anaesthetists and female dental assistants, but not their male counterparts in the USA, were reported to show an increased incidence of cancer (Cohen et al. 1971). Animal experiments showed variable results when exposed to anaesthetics by oral lavage or inhalation (Basford and Fink 1968, Coate et al. 1979, Harper et al. 1982). Reports of foetal wastage among anaesthetists have been consistent (Vessey 1978, Corbett et al. 1974, Cohen et al. 1971). The incidence of spontaneous abortions (Cohen et al. 1971, Knill-Jones et al. 1972, 1975), congenital malformation and infertility have also been reported among female anaesthetists and wives of male anaesthetists.

At the cellular level, anaesthetics inhibit the mitotic spindle and produce c-metaphases (Sturrock and Nunn 1975, 1976), and non-disjunction in Drosophila (Clements and Todd 1981). Halothane showed weak mutagenic activity in long term treatments of Drosophila, showing a slight increase in mutation frequency (Kramers and Burm 1979, Kundomal and Baden 1985).

Cytogenetic reports on exposure to anaesthetics are few. Lamberti et al. 1989, Natarajan and Santhiya 1990, Karelova et al. 1992 and Padmavathi et al. 1995, consistently reported a significant increase in CAs in anaesthetists, and so is the finding in this study.

The significance of the present work lies in the fact that the subject sample was composed entirely of anaesthetists, the exposure time varying from 2 years to 29 years. This is important as anaesthetists are stationed close to the apparatus and therefore have the highest exposure (Gardner 1989). In the work of previous workers, even though the subject sample (anesthetists, nurses and technicians) had variable exposures to the AGs (Table 3), there was a higher level of CAs in subjects (Lamberti et al. 1981, Natarajan and Santhiya 1992, Karelova et al. 1992, Padmavathy et al. 1995). Holmberg et al. (1982), however had only one anaesthetist in his sample, which may account

Table 3. Showing CA values

\begin{tabular}{|c|c|c|}
\hline Author, year, sample chosen & $\mathrm{CA}$ values & Conclusion \\
\hline \multicolumn{3}{|l|}{ Holmberg et al. (1982) } \\
\hline 11 subjects, $(1)^{*}$ & 2.2 & NS \\
\hline 10 controls & 1.2 & \\
\hline \multicolumn{3}{|l|}{ Lamberti et al. (1989) } \\
\hline 30 subjects, (not specified)* & 3.07 & Significantly higher \\
\hline 15 controls & 2.06 & \\
\hline \multicolumn{3}{|l|}{ Natarajan and Santhiya (1992) } \\
\hline 24 subjects, $(11)^{*}$ & mean values not provided & Significantly higher \\
\hline \multicolumn{3}{|l|}{24 controls } \\
\hline \multicolumn{3}{|l|}{ Karelova et al. (1992) } \\
\hline 24 subjects, $(14)^{*}$ & 3.84 & Significantly higher \\
\hline 30 controls & 1.87 & \\
\hline \multicolumn{3}{|l|}{ Padmavathi et al. (1995) } \\
\hline 36 subjects, $(20)^{*}$ & 2.46 & Significantly higher \\
\hline 20 controls & 1.60 & \\
\hline \multicolumn{3}{|l|}{ Present work (1996) } \\
\hline 51 subjects, $(51)^{*}$ & $4.29 \pm 2.69$ & Significantly higher \\
\hline 51 controls & $1.55 \pm 1.54$ & \\
\hline
\end{tabular}

* The numbers in parentheses ( ) indicate the number of anaesthetists in the sample. 
for his conclusion that CAs are not significantly higher in exposed personnel.

Another significant feature is that each subject was matched $1: 1$ with controls of the same sex, age ( \pm 1 year), diet, smoking habits (even to the number of cigarettes smoked/day), and general lifestyle (practicing physicians). This was aimed at reducing the known confounding variables.

Chromosomal aberrations such as gaps, breaks, deletions, inversions and other structural rearrangements are the consequence of breaks in the DNA due to a mutagenic agent such as ionizing radiation or a chemical agent (anesthetics in this case). Chemical mutagens react with DNA, RNA and protein to form covalent adducts and cross-linking, which are primary events in mutagenesis and carcinogenesis (Sankaranarayanan 1994). Dimers formed between adjacent pyrimidines are the lesions that are ultimately translated into structural aberrations. These occur at specific sites, often referred to as 'hot-spots', usually located in the G-light chromosome bands (Chandley 1991).

Single-strand (SS) breaks are repaired normally, while base damages are repaired slowly (Natarajan 1984). The various enzymes routinely present in the cell, normally a) repair the breaks by joining the broken ends (restitution or healing) or b) there may be misrepair if there are several broken ends resulting in inversions and other structural rearrangements, or c) no repiar at all resulting in gaps, breaks and delations.

The effect of the mutagen on the chromosome depends on the phase of the cell at the time of exposure. If the exposure is just prior to the S-phase, (late $G_{1}$ ) or in the $G_{2}$ phase, the lesions are of the chromatid-type, confirming the split nature of the chromosomes after synthesis. Early $\mathrm{G}_{1}$ insults result in the chromosome-type aberrations.

Chromosome aberrations were evaluated after $48 \mathrm{hr}$ of culturing, as it has been reported (Evans and O'Riordan 1975) that most cells have completed the first mitotic division by this time. Analysis after $72 \mathrm{hr}$ may show less CAs as repair processes may have taken place in the subsequent cell divisions. A decrease in breaks is associated with time due to repair mechanisms (Bryant and Slijepcevic 1993). The additional procedure of using the FPG stain helps to differentiate cells which have undergone more than 1 mitotic cycle even at $48 \mathrm{hr}$. Human peripheral lymphocytes are therefore particularly 'felicitous' for research purposes, as they can store the effects of damage until they are stimulated to undergo mitosis in culture (Wolff 1984).

The results obtained in the present work showed a significant difference of CAs in exposed subjects and unexposed controls. There was a marked increase of chromosomal aberrations like chromatid gaps and breaks, chromosome gaps, dicentrics, fragments and double minutes. Gaps have been included in this analysis, for even though gaps may not indicate true discontinuity, these are areas of despiralization which appear less well-stained (Therman 1986). Despiralization however represents a lesion, a structural modification, with loss of histone H1 protein and/or DNA, and this may be a response to a genotoxic insult. In this context, gaps assume significance.

The presence of chromatid breaks indicate that the damage was inflicted during the $G_{2}$ phase of the cell cycle and is consistent with reports of chemically induced damages (Therman 1986). Since circulating lymphocytes are in the $\mathrm{G}_{0}$ phase of its life cycle, it is assumed that the effect of the mutagen (AGs), produces aberrations during the $\mathrm{S}$ phase, probably due to misreplication. In vivo exposure to mutagens produces DNA lesions in lymphocytes which are repaired by cellular repair enzymes. Unrepaired lesions give rise to the chromatid type of aberrations (Natarajan 1993). Unrepaired lesions also indicate that the cells are inherently repair-deficient-a pre-requisite in the production of cancer (Jaiswal et al. 1994). Almost all tumors in their advanced stages show chromosomal changes - either gain or loss (monosomy or trisomy) partial gain or loss (duplication, amplification, or deletion) or rearrangements (translocations and inversions), which alter the number, positional relationships, regulation (activation) or the integrity of genes. These changes may activate oncogenes as shown by familial colorectal cancer (Review by Cohen et al. 1993). It is therefore important not to ignore the significance of an increase in chromosomal aberrations in the anaesthetists as a consequence of exposure. 


\section{Conclusion and recommendation}

Cytogenetic evaluation of 51 anaesthetists, presently working in Bangalore showed a significantly higher value of chromosomal aberrations like gaps, breaks, dicentrics, fragments and double minutes than that seen in matched controls. This suggests that the chronic exposure by anaesthetists to low levels of anaesthetic gases that are ambient in the operation theater is capable of producing chromosomal aberrations in the peripheral lymphocytes of anaesthetists, which may predispose to cancer. It is suggested that there is a strong case for the urgent introduction of effective scavenging devices to reduce the level of ambient gas in the theater, and to reduce the occupational hazard that the gas poses to the health of all the personnel in the operation room.

\section{References}

Arakaki, D. T. and Sparkes, R. S. 1963. Microtechnique for culturing leucocytes from whole blood. Cytogenetics 2: 57-60. Baden, J. M. and Simmons, V. F. 1980. Mutagenic effects of inhalational anesthetics. Mutat. Res. 75: 169-189.

Basford, A. B. and Fink, B. R. 1968. The teratogenicity of halothane in the rat. Anesthesiology 29: 1167-1173.

Bloom, A. D., Neriishi, S., Kamada, N., Iseki, T. and Keehn, R. J. 1966. Cytogenetic investigation of survivors of the atomic bombings of Hiroshima and Nagasaki. Lancet Sept. 24: 672-674.

Bryant, P. E. and Slijepcevic 1993. $\mathrm{G}_{2}$ chromatid aberations: Kinetics and possible mechanisms. Environ. Molec. Mutag. 22: $250-256$.

Chandley, A. C. 1991. On the parental origin of de novo mutation in man. J. Med. Gen. 28: 217-223.

Clements, J. and Todd, N. K. 1981. Halothane and non-disjunction in drosophila. Mutat. Res. 91: 225-228.

Coate, W. B., Kapp, R. W. and Lewis, T. R. 1979. Chronic exposure to low concentrations of halothane-nitrous oxide. Reproductive and cytogenetic effects in the rat. Anesthesiology 50: 310-318.

Cohen, E. N., Bellville, J. W. and Brown, B. W. 1971. Anesthesia, Pregnancy and Marriage. A study of operation room nurses and anesthetists. Anesthesiology 35: 343-347.

Cohen, M. M., Rosenblum-Vos, L. S. and Prabhakar, G. 1993. Human Cytogenetics. American Journal of Diseases in children 147: 1159-1166.

Corbett, T. H., Cornell, R. S., Endres, J. L. and Leiding, K. 1974. Birth defects among children of nurse-anesthetists. Anesthesiology 41: 341-344.

Evans, H. J. and O'Riordan, M. L. 1975. Human peripheral blood lymphocytes for the analysis of chromosome aberrations in mutagen tests. Mutat. Res. 31: 135-148.

Gardener, R. J. 1989. Inhalation Anaesthetics-Exposure and control: A statistical comparison of personal exposures in operating theaters with and without gas scavenging. Annals of Occupational Hygiene 33: 159-173.

Harper, M. H., Collins, P., Johnson, B. and Eger II, E. I. 1982. Hepatic injury following halothane, enflurane and isoflurane anesthesia in rats. Anesthesiology 56: 14-17.

Holmberg, K., Lambert, B., Lindsten, J. and Soderhall, S. 1982. DNA and chromosomal alterations in operating room personnel and in patients, before and after inhalation anaesthesia. Acta Anaesthesia Scandinavia 26: 531-539.

ISCN 1985. An International System for Human Cytogenetic Nomenclature. New York, Karger 1985.

Jaiswal, M., Anuradha, G., Rajeshwari, N. et al. 1994. Comet assay on cervical epithelial cells and leucocytes of patients with precancerous and cancerous lesions of the cervix. Med. Sci. Res. 22: 879-881.

Karelova, J., Jablonicka, A., Gavora, J. and Hano, L. 1992. Chromosome and sister-chromatid exchange analysis in peripheral lymphocytes and mutagenicity of urine in anesthesiology personnel. International Archives of Occupational and Environmental Health 64: 303-306.

Knill-Jones, R. P., Rodrigues, L. V., Moir, D. D. and Spence, A. A. 1972. Anaesthetic Practice and Pregnancy: Controlled survey of women anaesthetists in the UK. Lancet June 17: 1326-1328.

-, Newman, B. J. and Spence, A. A. 1975. Anaesthetic Practice and Pregnancy: Controlled survey of male anaesthetists. Lancet Oct. 25:

Kramers, P. G. N. and Burm, A. G. L. 1979. Mutagenicity studies with halothane in Drosophila melanogaster. Anesthesiology 50: $510-513$.

Kundomal, Y. R. and Baden, J. M. 1985. Mutagenicity of inhaled anesthetics in Drosophila melanogaster. Anesthesiology 62: 305-309.

Lamberti, L., Bigatti, P., Ardito, G. and Armellino, F. 1989. Chromosome analysis in operating room personnel. Mutagenesis 4: 95-97.

McNamee, R., Keen, R. I. and Corkill, C. M. 1987. Morbidity and early retirement among anaesthetists and other specialists. Anaesthesia 42: 133-140. 
Natarajan, A. T. 1993. Mechanism for induction of mutations and chromosome alterations. Environmental Health Perspectives 101 (Suppl. 3): 225-229.

- 1984. Origin and significance of chromosomal alterations. Mutations in Man. Ed. Obe, G.

Natrajan, D. and Santhiya, S. T. 1990. Cytogenetic damage in operation room personnel. Anaesthesia 45: 574-577.

Padmavathi, P., Aruna Prabhavathi, P., Hema Prasad, M., Fatima, S. K. and Reddy, P. P. 1995. Chromosomal aberrations in operating theater staff. Med. Sci. Res. 23 (4): 279-280.

Perry, P. and Wolff, S. 1974. New Giemsa method for the differential staining of sister chromatids. Nature 251: 156-158.

Sankaranarayanan, K. 1994. Estimation of genetic risks of exposure to chemical mutagens: Relevance of data on spontaneous mutations and of experience with ionizing radiation. Mutat. Res. 304: 117-120.

Spierdijk, J. and Burm, A. G. L. 1982. Environmental hazards in the operation theater associated with small amounts of anaesthetic agents. In chap. 57, Section V of Anaesthesia, 3rd ed., Eds. Scurr and Feldman, Wm Heinemann Medical Books Ltd., London.

Sturrock, J. E. and Nunn, J. F. 1975. Mitosis in mammalian cells during exposure to anesthetics. Anesthesiology 43: 21-23.

- and - 1976. Synergism between halothane and nitrous oxide in the production of nuclear abnormalities in the dividing fibroblast. Anesthesiology 44: 461-471.

Therman, E. 1986. In: Human Chromosomes-Structure, Behaviour, Effects. 2nd ed., Springer Verlag.

Vessey, M. P. 1978. Epidemiological studies of the occupational hazards of anaesthesia-A review. Anaesthesia 33: 430-438.

Wolff, S. 1984. Cytogenetics and environmental mutagens. In: Genetics: New Frontiers, Vol. III, Genetics and Health. Eds. Chopra, Joshi, Sharma and Bansal. 